data collection. $77.4 \%$ reported having presented at least one injury during the 12 preceding months. Higher mean training duration per week and some parameters of the final run-up (higher speed, higher contact time) and take-off (lower height at impulse) phases of the pole vault were significantly associated with history of all injuries.

Conclusions Although the design of the present study do not allow to determine the cause-consequence relationships regarding the biomechanical patterns and the injury occurrence, this study presents some interesting findings supporting the hypothesis of a relationship between the biomechanics pole vault pattern and the injury risk, which can be helpful in injury prevention perspective.

\section{HIGH BODY FAT ALTERS THROWING SHOULDER KINETICS IN SOFTBALL PITCHERS: IMPLICATIONS FOR INJURY PREVENTION}

Kenzie Friesen, Nicole Bordelon, Jessica Downs, Kyle Wasserberger, Gretchen Oliver. Auburn University, Auburn, USA

\subsection{6/bjsports-2021-IOC. 186}

Background Softball pitching is a strenuous task that accrues high forces at the shoulder resulting in frequent overuse injury, potentially exacerbated by increased pitcher body fat.

Objective To examine differences in injury rate, pain prevalence, and throwing shoulder kinetics between pitchers with high and healthy body fat percentage (BF\%). We hypothesized pitchers with high BF\% would display increased kinetics and higher rates of injury and pain.

Design Cross-sectional; retrospective.

Setting Indoor biomechanics laboratory with high-school league softball pitchers.

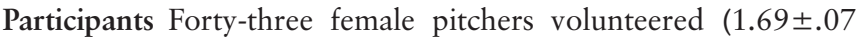
$\mathrm{m}, 75.97 \pm 16.85 \mathrm{~kg}, 15.2 \pm 1.2$ years) and were analyzed. Participants were currently on team rosters and surgery/injury free for the last six months.

Assessment of Risk Factors BF\% was determined using dualenergy $\mathrm{x}$-ray absorptiometry and pitchers were grouped into healthy $(<32 \mathrm{BF} \% ; \mathrm{n}=17)$ and high-fat $(\geq 32 \mathrm{BF} \% ; \mathrm{n}=26)$ categories.

Main Outcome Measurements Throwing shoulder distraction, superior, lateral, and anterior forces (normalized to body weight * pitch velocity) were measured via an electromagnetic motion capture system. History of softball related injuries and the presence of pain was self-reported on a health history questionnaire.

Results $83.3 \%$ of pitchers currently experiencing pain (total $\mathrm{n}=12$ ) and $72.2 \%$ of pitchers who had suffered a softballrelated injury (total $\mathrm{n}=18$ ) were high-fat pitchers. Statistical parametric mapping was used to determine differences in throwing shoulder forces between groups during foot contact to follow-through of the pitch. Analyses revealed high-fat pitchers had higher normalized distraction force over most of the first $27 \%$ of the pitch $\left(t_{\text {crit }}=3.112, p<.008\right)$ and lower normalized superior force during $80-91 \%$ of the pitch $\left(\mathrm{t}_{\text {crit }}=3.141, \mathrm{p}=.004\right)$.

Conclusions High BF\% alters shoulder kinetics during the pitch and may increase high-school softball pitchers' injury risk. Athletes, coaches, and clinicians should understand this increased risk and safely emphasize the importance of attaining a healthy body composition.

\section{VARIABLES ASSOCIATED WITH KNEE VALGUS IN MALE SOCCER PLAYERS DURING A SINGLE LEG VERTICAL LANDING TASK}

${ }^{1}$ Gustavo Leporace, ${ }^{1}$ Matheus Vianna, ${ }^{1}$ Leonardo Metsavaht, ${ }^{2}$ Marcio Tannure, ${ }^{3}$ Alex Souto Maior. 'Institute Brazil of Technologies in Health, RJ, Brazil; ${ }^{2}$ Performance Excellence Center, Flamengo Sport Club, RJ, Brazil; ${ }^{3}$ Department of Rehabilitation Sciences, University Augusto Mota, RJ, Brazil

\subsection{6/bjsports-2021-IOC.187}

Background Knee valgus during landing and cutting situations has been described as the main biomechanical risk factor for anterior cruciate ligament (ACL) injuries. Evidence support the influence of hip strength and biomechanics on knee valgus in female athletes. To develop specific preventive measures for males, it is important to understand biomechanical and musculoskeletal variables associated with this mechanism during activities that mimic ACL injuries.

Objective To test the correlation between peak knee valgus during a single-leg vertical jump-landing task, and biomechanical and musculoskeletal variables in male soccer professional athletes.

Design Cross-sectional study.

Setting Biomechanics laboratory.

Participants Twenty-four healthy male professional soccer players.

Interventions Participants performed a vertical jump-landing task during the pre-season. Kinematic data were collected using an 8 high-speed cameras motion analysis system (Vicon, Oxford, UK) with a sample rate of $250 \mathrm{~Hz}$. Maximal isometric hip strength was tested using a hand-held dynamometer and ankle dorsiflexion range of motion was measured using a digital inclinometer.

Main Outcome Measurements Predictor variables were lateral trunk lean, hip adduction and internal rotation, hip extensors, abductor and external rotator isometric strength, and ankle dorsiflexion range of motion. Interest variables were peak knee valgus during the landing task. Association between predictors and interest variables were tested using Pearson Correlation Coefficient (alpha $=0.05$ ).

Results Lateral trunk lean $(\mathrm{r}=0.43, \mathrm{p}=0.04)$ and hip internal rotation $(\mathrm{r}=-0.68, \mathrm{p}<0.01)$ showed significant correlation with peak knee valgus during the vertical landing task.

Conclusions Trunk position seems to influence the ACL mechanism, possibly by increasing knee abduction moment related to the lateral displacement of centre of mass during lateral trunk lean. The negative correlation between hip internal rotation and knee valgus in males is opposite to the results usually described for females, and may be related to the different influence of hip on ACL injury mechanism between sexes.

\section{ABSTRACT WITHDRAWN}

